\title{
Weyl type theorem for operator matrices
}

\author{
by \\ XiaOhong CaO (Xi'an)
}

\begin{abstract}
Using topological uniform descent, we give necessary and sufficient conditions for Browder's theorem and Weyl's theorem to hold for an operator $A$. The two theorems are liable to fail for $2 \times 2$ operator matrices. In this paper, we explore how they survive for $2 \times 2$ operator matrices on a Hilbert space.
\end{abstract}

1. Introduction. Throughout this note, let $H$ and $K$ be complex, separable, infinite-dimensional Hilbert spaces, and let $B(H, K)$ denote the set of bounded linear operators from $H$ to $K$; we abbreviate $B(H, H)$ to $B(H)$. If $A \in B(H)$, write $N(A)$ and $R(A)$ for the null space and range of $A ; \sigma(A)$ for the spectrum of $A ; \varrho(A)=\mathbb{C} \backslash \sigma(A) ; \pi_{00}(A)=\pi_{0}(A) \cap$ iso $\sigma(A)$, where $\pi_{0}(A)=\{\lambda \in \mathbb{C}: 0<\operatorname{dim} N(A-\lambda I)<\infty\}$ are the eigenvalues of finite multiplicity. An operator $A \in B(H)$ is called upper semi-Fredholm if it has closed range with finite-dimensional null space; if $R(A)$ has finite codimension, then $A \in B(H)$ is called lower semi-Fredholm. We call $A \in B(H)$ Fredholm if it is both upper and lower semi-Fredholm. If $A$ is upper or lower semi-Fredholm, then the index of $A$, ind $(A)$, is defined to be $\operatorname{ind}(A)=\operatorname{dim} N(A)-\operatorname{dim} H / R(A)$. The ascent of $A, \operatorname{asc}(A)$, is the least nonnegative integer $n$ such that $N\left(A^{n}\right)=N\left(A^{n+1}\right)$, and the descent, $\operatorname{des}(A)$, is the least nonnegative integer $n$ such that $R\left(A^{n}\right)=R\left(A^{n+1}\right)$. The operator $A$ is Weyl if it is Fredholm of index zero, and Browder if it is Fredholm of finite ascent and descent. The essential spectrum $\sigma_{\mathrm{e}}(A)$, Weyl spectrum $\sigma_{\mathrm{w}}(A)$ and Browder spectrum $\sigma_{\mathrm{b}}(A)$ of $A$ are defined by

$$
\begin{aligned}
\sigma_{\mathrm{e}}(A) & =\{\lambda \in \mathbb{C}: A-\lambda I \text { is not Fredholm }\}, \\
\sigma_{\mathrm{w}}(A) & =\{\lambda \in \mathbb{C}: A-\lambda I \text { is not Weyl }\}, \\
\sigma_{\mathrm{b}}(A) & =\{\lambda \in \mathbb{C}: A-\lambda I \text { is not Browder }\} .
\end{aligned}
$$

2000 Mathematics Subject Classification: 47A15, 47A53, 47A55.

Key words and phrases: Weyl's theorem, Browder's theorem, topological uniform descent.

This work is supported by the NSF of China (No. 10726043) and by the Support Plan of Education Ministry, P.R. China, for Talented Persons of the New Century. 
Let $\sigma_{\mathrm{a}}(A)$ denote the approximate point spectrum of the operator $A \in B(H)$ and set $\pi_{00}^{\mathrm{a}}(A)=\pi_{0}(A) \cap$ iso $\sigma_{\mathrm{a}}(A)$. We say that Browder's theorem holds for $A$ if

$$
\sigma_{\mathrm{w}}(A)=\sigma_{\mathrm{b}}(A)
$$

Weyl's theorem holds for $A$ if

$$
\sigma(A) \backslash \sigma_{\mathrm{w}}(A)=\pi_{00}(A),
$$

and $a$-Weyl's theorem holds for $A$ if

$$
\sigma_{\mathrm{a}}(A) \backslash \sigma_{\mathrm{ea}}(A)=\pi_{00}^{\mathrm{a}}(A),
$$

where $\sigma_{\text {ea }}(A)=\left\{\lambda \in \mathbb{C}: A-\lambda I \notin \mathrm{SF}_{+}^{-}(H)\right\}$ and $\mathrm{SF}_{+}^{-}(H)=\{A \in B(H)$ : $A$ is upper semi-Fredholm of $\operatorname{ind}(A) \leq 0\}$. Browder's essential approximate point spectrum $\sigma_{\mathrm{ab}}(A)$ is defined by $\sigma_{\mathrm{ab}}(A)=\sigma_{\mathrm{ea}}(A) \cup \mathrm{acc} \sigma_{\mathrm{a}}(A)$. If $\sigma_{\mathrm{ab}}(A)=$ $\sigma_{\text {ea }}(A)$, we say that a-Browder's theorem holds for $A$. The concept of aWeyl's theorem was introduced by Rakočević: a-Weyl's theorem for $A \Rightarrow$ Weyl's theorem for $A$, but the converse is generally false [9].

Sufficient conditions for an operator $A \in B(H)$ to satisfy Weyl's theorem have recently been considered by a number of authors ([1], [3], [7], e.g.). The plan of this paper is as follows. In Section 2, we prove our main result and give necessary and sufficient conditions for Weyl's theorem and Browder's theorem to hold for $A$. In Section 3, we explore how the two theorems survive for $2 \times 2$ operator matrices.

2. Weyl type theorem and topological uniform descent. If $A \in B(H)$, then for each nonnegative integer $n, A$ induces a linear transformation from the vector space $R\left(A^{n}\right) / R\left(A^{n+1}\right)$ to $R\left(A^{n+1}\right) / R\left(A^{n+2}\right)$. We will let $k_{n}(A)$ be the dimension of the null space of the induced map and let $k(A)=\sum_{n=0}^{\infty} k_{n}(A)$. The following definition describes the classes of operators we will study. These definitions were introduced by S. Grabiner [5].

Definition 2.1. If there is a nonnegative integer $d$ for which $k_{n}(A)=0$ for $n \geq d$ (i.e., the induced maps are isomorphisms for $n \geq d$ ), we say that $A$ has uniform descent for $n \geq d$.

Definition 2.2. Suppose there is a nonnegative integer $d$ such that $A$ has uniform descent for $n \geq d$. If $R\left(A^{n}\right)$ is closed in the operator range topology of $R\left(A^{d}\right)$ for $n \geq d$, then we say that $A$ has topological uniform descent.

It can be shown that if $A$ is upper semi-Fredholm, then $A$ has topological uniform descent. Let

$$
\varrho_{\tau}(A)=\{\lambda \in \mathbb{C}: A-\lambda I \text { has topological uniform descent }\} .
$$


If $A$ has topological uniform descent, then it has the following property ([5, Corollary 4.9]):

Lemma 2.3. Suppose that $A \in B(H)$ and $\lambda$ belongs to the boundary of the spectrum of $A$. If $A-\lambda I$ has topological uniform descent, then $\lambda$ is a pole of $A$.

Let

$\varrho_{1}(A)=\{\lambda \in \mathbb{C}: \operatorname{dim} N(A-\lambda I)<\infty$ and there exists $\varepsilon>0$ such that

$$
A-\mu I \text { is Weyl if } 0<|\mu-\lambda I|<\varepsilon\},
$$

and let $\sigma_{1}(A)=\mathbb{C} \backslash \varrho_{1}(A)$. Clearly, $\sigma_{1}(A) \subseteq \sigma_{\mathrm{w}}(A)$.

Theorem 2.4. Browder's theorem holds for $A \Leftrightarrow \varrho_{\tau}(A) \subseteq \varrho_{\mathrm{b}}(A) \cup \sigma_{1}(A)$, where $\varrho_{\mathrm{b}}(A)=\mathbb{C} \backslash \sigma_{\mathrm{b}}(A)$.

Proof. Suppose $\varrho_{\tau}(A) \subseteq \varrho_{\mathrm{b}}(A) \cup \sigma_{1}(A)$. If $A-\lambda I$ is Weyl, then $\lambda \in \varrho_{\tau}(A)$. Hence $\lambda \in \varrho_{\mathrm{b}}(A) \cup \sigma_{1}(A)$. Since $\lambda \notin \sigma_{1}(A)$, it follows that $\lambda \in \varrho_{\mathrm{b}}(A)$, which means that $A-\lambda I$ is Browder. Thus $\sigma_{\mathrm{w}}(A)=\sigma_{\mathrm{b}}(A)$, and therefore Browder's theorem holds for $A$.

Conversely, suppose Browder's theorem holds for $A$. Let $\lambda_{0} \in \varrho_{\tau}(A)$. If $\lambda_{0} \notin \sigma_{1}(A)$, then $\operatorname{dim} N\left(A-\lambda_{0} I\right)<\infty$ and there exists $\varepsilon>0$ such that $A-\lambda I$ is Weyl if $0<\left|\lambda-\lambda_{0}\right|<\varepsilon$. Since Browder's theorem holds for $A$, it follows that $A-\lambda I$ is Browder. Hence $\lambda_{0} \in \varrho(A) \cup \partial \sigma(A)$. Without loss of generality, we suppose $\lambda_{0} \in \partial \sigma(A)$. By Lemma $2.3, \lambda_{0}$ is a pole of $A$. Let $\operatorname{asc}\left(A-\lambda_{0} I\right)=\operatorname{des}\left(A-\lambda_{0} I\right)=p$. Then $H=N\left[\left(A-\lambda_{0} I\right)^{p}\right] \oplus R\left[\left(A-\lambda_{0} I\right)^{p}\right]$. Using the fact that $\operatorname{dim} N\left(A-\lambda_{0} I\right)<\infty$, we infer that $A-\lambda_{0} I$ is Browder. Therefore $\lambda_{0} \in \varrho_{\mathrm{b}}(A)$. This proves $\varrho_{\tau}(A) \subseteq \varrho_{\mathrm{b}}(A) \cup \sigma_{1}(A)$.

Theorem 2.5. Weyl's theorem holds for $A \Leftrightarrow \pi_{00}(A) \subseteq \varrho_{\tau}(A) \subseteq$ $\varrho_{\mathrm{b}}(A) \cup \sigma_{1}(A)$.

Proof. Suppose Weyl's theorem holds for $A$. Since Weyl's theorem implies Browder's theorem, from Theorem 2.4, $\varrho_{\tau}(A) \subseteq \varrho_{\mathrm{b}}(A) \cup \sigma_{1}(A)$. Let $\lambda_{0} \in \pi_{00}(A)$. Then $A-\lambda_{0} I$ is Browder because Weyl's theorem holds for $A$. Therefore $\lambda_{0} \in \varrho_{\tau}(A)$. Thus $\pi_{00}(A) \subseteq \varrho_{\tau}(A) \subseteq \varrho_{\mathrm{b}}(A) \cup \sigma_{1}(A)$.

For the converse, the condition $\varrho_{\tau}(A) \subseteq \varrho_{\mathrm{b}}(A) \cup \sigma_{1}(A)$ implies that Browder's theorem holds for $A$, that is, $\sigma(A) \backslash \sigma_{\mathrm{w}}(A) \subseteq \pi_{00}(A)$. If $\lambda_{0} \in$ $\pi_{00}(A)$, then $\lambda_{0} \in \varrho_{\tau}(A) \subseteq \varrho_{\mathrm{b}}(A) \cup \sigma_{1}(A)$. Since $\lambda_{0} \notin \sigma_{1}(A)$, it follows that $\lambda_{0} \in \varrho_{\mathrm{b}}(A)$. Hence $A-\lambda_{0} I$ is Browder, which means that $\lambda_{0} \in \sigma(A) \backslash \sigma_{\mathrm{w}}(A)$. Thus $\sigma(A) \backslash \sigma_{\mathrm{w}}(A)=\pi_{00}(A)$, and so Weyl's theorem holds for $A$.

Corollary 2.6. Weyl's theorem holds for $A$ and $A^{*} \Leftrightarrow\left[\pi_{00}(A) \cup \pi_{00}\left(A^{*}\right)\right]$ $\subseteq \varrho_{\tau}(A) \subseteq \varrho_{\mathrm{b}}(A) \cup \sigma_{1}(A)$.

Let $H(A)$ be the class of all complex-valued functions which are analytic on a neighborhood of $\sigma(A)$ and are not constant on any component of $\sigma(A)$. 
Corollary 2.7. Suppose iso $\sigma(A) \subseteq \varrho_{\tau}(A) \subseteq \varrho_{\mathrm{b}}(A) \cup \sigma_{1}(A)$. Then:

(1) Weyl's theorem holds for $A$ and $A^{*}$.

(2) If $f \in H(A)$, then Weyl's theorem holds for $f(A)$ and $f(A)^{*}$ if and only if Browder's theorem holds for $f(A)$.

Let

$\varrho_{2}(A)=\{\lambda \in \mathbb{C}: \operatorname{dim} N(A-\lambda I)<\infty$ and there exists $\varepsilon>0$ such that

$$
\left.A-\mu I \in \mathrm{SF}_{+}^{-}(H) \text { if } 0<|\mu-\lambda I|<\varepsilon\right\},
$$

and let $\sigma_{2}(A)=\mathbb{C} \backslash \varrho_{2}(A)$. Clearly, $\sigma_{2}(A) \subseteq \sigma_{1}(A)$.

Corollary 2.8. Suppose iso $\sigma(A) \subseteq \varrho_{\tau}(A) \subseteq \varrho_{\mathrm{b}}(A) \cup \sigma_{2}(A)$. Then:

(1) Weyl's theorem holds for $A$ and $A^{*}$.

(2) a-Weyl's theorem holds for A.

(3) Weyl's theorem holds for $f(A)$ and $f(A)^{*}$ for any $f \in H(A)$.

(4) $a$-Weyl's theorem holds for $f(A)$ for any $f \in H(A)$.

Proof. (1) Apply Corollary 2.7.

(2) Since iso $\sigma(A) \subseteq \varrho_{\tau}(A) \subseteq \varrho_{\mathrm{b}}(A) \cup \sigma_{2}(A)$, it follows that $\sigma(A)=$ $\sigma_{\mathrm{a}}(A)$ and $\sigma_{\mathrm{w}}(A)=\sigma_{\mathrm{ea}}(A)=\sigma_{\mathrm{b}}(A)$. Weyl's theorem for $A$ implies that $\sigma(A) \backslash \sigma_{\mathrm{w}}(A)=\pi_{00}(A)$. Thus $\sigma_{\mathrm{a}}(A) \backslash \sigma_{\mathrm{ea}}(A)=\pi_{00}^{\mathrm{a}}(A)$, which means that a-Weyl's theorem holds for $A$.

(3) Let $\mu_{0} \in \sigma(f(A)) \backslash \sigma_{\mathrm{w}}(f(A))$ and let

$$
f(A)-\mu_{0} I=a\left(A-\lambda_{1} I\right)^{n_{1}} \cdots\left(A-\lambda_{k} I\right)^{n_{k}} g(A),
$$

where $\lambda_{i} \neq \lambda_{j}, a \neq 0$ and $g(A)$ is invertible. Then $A-\lambda_{i} I$ is Fredholm and $\sum_{i=1}^{k} \operatorname{ind}\left[\left(A-\lambda_{i} I\right)^{n_{i}}\right]=\operatorname{ind}\left(f(A)-\mu_{0} I\right)=0$. Hence $\lambda_{i} \in \varrho_{\tau}(A) \subseteq$ $\varrho_{\mathrm{b}}(A) \cup \sigma_{2}(A)$. Suppose $\lambda_{1}, \ldots, \lambda_{j} \in \varrho_{\mathrm{b}}(A)$ and $\lambda_{j+1}, \ldots, \lambda_{k} \in \sigma_{2}(A)$. Using the definition of $\varrho_{2}(A)$, we deduce that $\operatorname{ind}\left(A-\lambda_{i} I\right)>0$ for $i=j+1, \ldots, k$. Thus

$$
\begin{aligned}
\sum_{i=1}^{k} \operatorname{ind}\left[\left(A-\lambda_{i} I\right)^{n_{i}}\right] & =\sum_{i=1}^{j} \operatorname{ind}\left[\left(A-\lambda_{i} I\right)^{n_{i}}\right]+\sum_{i=j+1}^{k} \operatorname{ind}\left[\left(A-\lambda_{i} I\right)^{n_{i}}\right] \\
& =0+\sum_{i=j+1}^{k} \operatorname{ind}\left[\left(A-\lambda_{i} I\right)^{n_{i}}\right]>0 .
\end{aligned}
$$

This is a contradiction, so $\lambda_{i} \in \varrho_{\mathrm{b}}(A)$ for all $i=1, \ldots, k$. Therefore $f(A)-$ $\mu_{0} I$ is Browder, which means that $\sigma(f(A)) \backslash \sigma_{\mathrm{w}}(f(A)) \subseteq \pi_{00}(f(A))$. For the converse, let $\mu_{0} \in \pi_{00}(f(A))$ and let

$$
f(A)-\mu_{0} I=a\left(A-\lambda_{1} I\right)^{n_{1}} \cdots\left(A-\lambda_{k} I\right)^{n_{k}} g(A),
$$

where $\lambda_{i} \neq \lambda_{j}, a \neq 0$ and $g(A)$ is invertible. Without loss of generality, we suppose that $\lambda_{i} \in \sigma(A), i=1, \ldots, k$. Thus $\lambda_{i} \in$ iso $\sigma(A)$ and $\operatorname{dim} N\left(A-\lambda_{i} I\right)$ $<\infty$. Hence $\lambda_{i} \in \varrho_{\tau}(A) \subseteq \varrho_{\mathrm{b}}(A) \cup \sigma_{2}(A)$ and $\lambda_{i} \notin \sigma_{2}(A)$. Then $A-\lambda_{i} I$ 
is Browder. This implies that $f(A)-\mu_{0} I$ is Browder, that is, $\pi_{00}(f(A)) \subseteq$ $\sigma(f(A)) \backslash \sigma_{\mathrm{w}}(f(A))$. Hence $\sigma(f(A)) \backslash \sigma_{\mathrm{w}}(f(A))=\pi_{00}(f(A))$, which means that Weyl's theorem holds for $f(A)$.

(4) Since $\sigma(A)=\sigma_{\mathrm{a}}(A)$, it follows that $\sigma_{\mathrm{a}}(f(A))=f\left(\sigma_{\mathrm{a}}(A)\right)=f(\sigma(A))$ $=\sigma(f(A))$. Similar to the proof of (3), we can show that $\sigma_{\text {ea }}(f(A))=$ $\sigma_{\mathrm{b}}(f(A))$. Since Weyl's theorem holds for $f(A)$, it follows that $\sigma_{\mathrm{w}}(f(A))=$ $\sigma_{\mathrm{b}}(f(A))$. Thus $\sigma_{\text {ea }}(f(A))=\sigma_{\mathrm{b}}(f(A))=\sigma_{\mathrm{w}}(f(A))$. Now Weyl's theorem for $f(A)$ implies that a-Weyl's theorem also holds for $f(A)$.

ExAmple. Recall that $A \in B(H)$ is said to be $*$-paranormal if

$$
\left\|A^{*} x\right\|^{2} \leq\left\|A^{2} x\right\|\|x\| \quad \text { for all } x \in H,
$$

and totally $*$-paranormal if $A-\lambda I$ is $*$-paranormal for every $\lambda \in \mathbb{C}$. We have the following facts:

(1) If $A \in B(H)$ is totally $*$-paranormal, then $A-\lambda I$ has finite ascent for each $\lambda \in \mathbb{C}$.

(2) If $A \in B(H)$ is totally $*$-paranormal, then $H_{0}(A-\lambda I)=N(A-\lambda I)$ for every $\lambda \in \mathbb{C}$, where

$$
H_{0}(A-\lambda I)=\left\{x \in H: \lim _{n \rightarrow \infty}\left\|(A-\lambda I)^{n} x\right\|^{1 / n}=0\right\} .
$$

In fact, for each $\lambda \in \mathbb{C}$, if $x \in N\left[(A-\lambda I)^{2}\right]$, then $\left(A^{*}-\lambda I\right)(A-\lambda I) x=0$. It follows that $(A-\lambda I) x=0$, which means that $A-\lambda I$ has finite ascent for every $\lambda \in \mathbb{C}$.

Since any $*$-paranormal operator is normaloid, it follows that $\|(A-\lambda I) x\|$ $\leq\left\|(A-\lambda I)^{n} x\right\|^{1 / n}$ for all $x \in H$ and $n \in \mathbb{N}$, and hence $H_{0}(A-\lambda I) \subseteq$ $N(A-\lambda I)$. The converse inclusion is clear.

We next claim that:

(1) If $A \in B(H)$ is totally *-paranormal, then Weyl's theorem hold for $f(A)$ and $f(A)^{*}$ for every $f \in H(A)$.

(2) If $A^{*} \in B(H)$ is totally $*$-paranormal, then a-Weyl's theorem holds for $f(A)$ for every $f \in H(A)$.

For (1), from Corollary 2.7, we only need to prove that iso $\sigma(A) \subseteq$ $\varrho_{\tau}(A) \subseteq \varrho_{\mathrm{b}}(A) \cup \sigma_{1}(A)$ and that Browder's theorem holds for $f(A)$ for every $f \in H(A)$.

Let $\lambda_{0} \in$ iso $\sigma(A)$. Then there are decompositions $H=H_{0}\left(A-\lambda_{0} I\right) \oplus M$ and $A=A_{1} \oplus A_{2}$, where $\sigma\left(A_{1}\right)=\left\{\lambda_{0}\right\}$ and $A_{2}-\lambda_{0} I$ is invertible. Since $H_{0}\left(A-\lambda_{0} I\right)=N\left(A-\lambda_{0} I\right)$, it follows that $A_{1}-\lambda_{0} I=0$. Thus $A-\lambda_{0} I$ has finite ascent and finite descent, which means that $\lambda_{0}$ is a pole of $A$. Hence $\lambda_{0} \in \varrho_{\tau}(A)$. Next, suppose $\lambda_{0} \in \varrho_{\tau}(A) \backslash \sigma_{1}(A)$. Then $\operatorname{dim} N\left(A-\lambda_{0} I\right)<\infty$ and there exists $\varepsilon>0$ such that $A-\lambda I$ is Weyl if $0<\left|\lambda-\lambda_{0}\right|<\varepsilon$. Since $A-\lambda I$ has finite ascent, it follows that $A-\lambda I$ is Browder, which means that $\lambda_{0}$ is on the boundary of the spectrum of $A$ or $A-\lambda_{0} I$ is 
invertible. Thus $\lambda_{0} \in \varrho_{\mathrm{b}}(A)$, so we have proved that iso $\sigma(A) \subseteq \varrho_{\tau}(A) \subseteq$ $\varrho_{\mathrm{b}}(A) \cup \sigma_{1}(A)$. For any $f \in H(A)$, suppose $f(A)-\mu_{0} I$ is Weyl and let $f(A)-\mu_{0} I=\left(A-\lambda_{1} I\right)^{n_{1}} \cdots\left(A-\lambda_{k} I\right)^{n_{k}} g(A)$, where $\lambda_{i} \neq \lambda_{j}$ and $g(A)$ is invertible. Then $A-\lambda_{i} I$ is Fredholm and has finite ascent for each $i=$ $1, \ldots, k$. Thus $f(A)-\mu_{0} I$ has finite ascent, which means that $f(A)-\mu_{0} I$ is Browder. Then $\sigma_{\mathrm{w}}(f(A))=\sigma_{\mathrm{b}}(f(A))$, and hence Browder's theorem holds for $f(A)$ for every $f \in H(A)$.

For $(2)$, since $A^{*}$ is totally $*$-paranormal, it follows that $\sigma_{\mathrm{a}}(A)=\sigma(A)$ and $\sigma_{1}(A)=\sigma_{2}(A)$. We can then prove that iso $\sigma(A) \subseteq \varrho_{\tau}(A) \subseteq$ $\varrho_{\mathrm{b}}(A) \cup \sigma_{2}(A)$, so a-Weyl's theorem holds for $f(A)$ for every $f \in H(A)$.

3. Weyl type theorem for operator matrices. The study of upper triangular operator matrices arises naturally from the following fact: if $A$ is a Hilbert space operator and $M$ is an invariant subspace for $A$, then $A$ has the following $2 \times 2$ upper triangular operator matrix representation:

$$
A=\left(\begin{array}{ll}
* & * \\
0 & *
\end{array}\right): M \oplus M^{\perp} \rightarrow M \oplus M^{\perp},
$$

and one way to study operators is to see them as entries of simpler operators. The upper triangular operator matrices have been studied by many authors ([6], [8], e.g.). When $A \in B(H)$ and $B \in B(K)$ are given, we denote by $M_{C}$ an operator acting on $H \oplus K$ of the form

$$
M_{C}=\left(\begin{array}{cc}
A & C \\
0 & B
\end{array}\right)
$$

where $C \in B(K, H)$. If $C=0$, let $M_{0}=\left(\begin{array}{cc}A & 0 \\ 0 & B\end{array}\right)$.

Weyl's theorem may or may not hold for a direct sum of operators for which Weyl's theorem holds. In this section, using the new spectrum set $\sigma_{2}(\cdot)$ and the topological uniform descent, we explore the Weyl type theorem for $2 \times 2$ operator matrices. We begin with ([2, Lemma 3.1]):

Lemma 3.1. For a given pair $(A, B)$ of operators, if both $A$ and $B$ have finite ascent, then for every $C \in B(K, H), M_{C}$ has finite ascent.

Theorem 3.2. Let $A \in B(H)$ be such that $\varrho_{\tau}(A) \subseteq \varrho_{\mathrm{b}}(A) \cup \sigma_{2}(A)$ and let $B \in B(K)$.

(1) If Browder's theorem holds for $\left(\begin{array}{cc}A & C_{0} \\ 0 & B\end{array}\right)$ for some $C_{0} \in B(K, H)$, then it holds for $\left(\begin{array}{cc}A & C \\ 0 & B\end{array}\right)$ for every $C \in B(K, H)$.

(2) If a-Browder's theorem holds for $\left(\begin{array}{cc}A & C_{0} \\ 0 & B\end{array}\right)$ for some $C_{0} \in B(K, H)$, then it holds for $\left(\begin{array}{cc}A & C \\ 0 & B\end{array}\right)$ for every $C \in B(K, H)$. 
Proof. (1) For any $C \in B(K, H)$, if $M_{C}-\lambda_{0} I$ is Weyl, from

$$
M_{C}-\lambda_{0} I=\left(\begin{array}{cc}
I & 0 \\
0 & B-\lambda_{0} I
\end{array}\right)\left(\begin{array}{cc}
I & C \\
0 & I
\end{array}\right)\left(\begin{array}{cc}
A-\lambda_{0} I & 0 \\
0 & I
\end{array}\right)
$$

we know $A-\lambda_{0} I$ is upper semi-Fredholm, $B-\lambda_{0} I$ is lower semi-Fredholm and $A-\lambda_{0} I$ is Fredholm if and only if $B-\lambda_{0} I$ is Fredholm. Since $A-\lambda_{0} I$ is upper semi-Fredholm, $\lambda_{0} \in \varrho_{\tau}(A) \subseteq \varrho_{\mathrm{b}}(A) \cup \sigma_{2}(A)$. By the definition of $\varrho_{2}(A)$, we must have $\operatorname{ind}\left(A-\lambda_{0} I\right) \geq 0$, which means that $A-\lambda_{0} I$ is Fredholm. Then $B-\lambda_{0} I$ is Fredholm and hence $\operatorname{ind}\left(M_{C_{0}}-\lambda_{0} I\right)=\operatorname{ind}(A-$ $\left.\lambda_{0} I\right)+\operatorname{ind}\left(B-\lambda_{0} I\right)=\operatorname{ind}\left(M_{C}-\lambda_{0} I\right)=0$. Therefore $M_{C_{0}}-\lambda_{0} I$ is Weyl. But since Browder's theorem holds for $M_{C_{0}}$, it follows that $M_{C_{0}}-\lambda_{0} I$ is Browder. Thus $\operatorname{asc}\left(A-\lambda_{0} I\right)<\infty$ and $\operatorname{des}\left(B-\lambda_{0} I\right)<\infty$. This implies $\lambda_{0} \notin \sigma_{2}(A)$, which means that $A-\lambda_{0} I$ is Browder. Since both $M_{C_{0}}-\lambda_{0} I$ and $A-\lambda_{0} I$ are Browder and $B-\lambda_{0} I$ is Fredholm, it follows that $\lambda_{0} \in$ iso $\sigma(B) \cup \varrho(B)$, hence $B-\lambda_{0} I$ is Browder. Thus $M_{C}-\lambda_{0} I$ is Browder. Now we see that $\sigma_{\mathrm{w}}\left(M_{C}\right)=\sigma_{\mathrm{b}}\left(M_{C}\right)$, which means that Browder's theorem holds for $M_{C}$ for every $C \in B(K, H)$.

(2) For every $C \in B(K, H)$ if $M_{C}-\lambda_{0} I \in \mathrm{SF}_{+}^{-}(H \oplus K)$, then $A-\lambda_{0} I$ is upper semi-Fredholm. Thus $\lambda_{0} \in \varrho_{\tau}(A) \subseteq \varrho_{\mathrm{b}}(A) \cup \sigma_{2}(A)$. From the proof of (1) we know that $A-\lambda_{0} I$ is Fredholm. Theorem 2.1 in [2] tells us that $B-\lambda_{0} I$ is upper semi-Fredholm and $M_{C_{0}}-\lambda_{0} I \in \mathrm{SF}_{+}^{-}(H \oplus K)$. Since aBrowder's theorem holds for $M_{C_{0}}$, it follows that $M_{C_{0}}-\lambda_{0} I$ has finite ascent. Hence $A-\lambda_{0} I$ has finite ascent, which means that $A-\lambda_{0} I$ is Browder. Thus there exists $\varepsilon>0$ such that $M_{C_{0}}-\lambda I$ is bounded from below and $A-\lambda I$ is invertible if $0<\left|\lambda-\lambda_{0}\right|<\varepsilon$. This implies that $\lambda_{0} \in$ iso $\sigma_{\mathrm{a}}(B)$ or $B-\lambda_{0} I$ is bounded from below. But since $B-\lambda_{0} I$ is upper semi-Fredholm, it has finite ascent ([4, Theorem 11]). Then $M_{C}-\lambda_{0} I$ has finite ascent, which means that $\lambda_{0} \notin \sigma_{\mathrm{ab}}\left(M_{C}\right)$. Thus $\sigma_{\mathrm{ea}}\left(M_{C}\right)=\sigma_{\mathrm{ab}}\left(M_{C}\right)$ and hence a-Browder's theorem holds for $M_{C}$ for every $C \in B(K, H)$.

Corollary 3.3. If $\varrho_{\tau}(A) \subseteq \varrho_{\mathrm{b}}(A) \cup \sigma_{2}(A)$, then for every $B \in B(K)$ and $C \in B(K, H)$ :

(1) If Browder's theorem holds for $\left(\begin{array}{cc}A & 0 \\ 0 & B\end{array}\right)$, then it also does for $\left(\begin{array}{cc}A & C \\ 0 & B\end{array}\right)$.

(2) If a-Browder's theorem holds for $\left(\begin{array}{cc}A & 0 \\ 0 & B\end{array}\right)$, then it also does for $\left(\begin{array}{cc}A & C \\ 0 & B\end{array}\right)$.

REMARK. The implications of Theorem 3.2 may fail for Weyl's theorem and a-Weyl's theorem. To see this, let $A, B, C \in B\left(\ell_{2}\right)$ be defined by

$$
\begin{aligned}
& A\left(x_{1}, x_{2}, x_{3}, \ldots\right)=\left(0, x_{1}, 0, \frac{1}{2} x_{2}, 0, \frac{1}{3} x_{3}, \ldots\right), \\
& B\left(x_{1}, x_{2}, x_{3}, \ldots\right)=\left(0, x_{2}, 0, x_{4}, \ldots\right), \\
& C\left(x_{1}, x_{2}, x_{3}, \ldots\right)=\left(x_{1}, 0,0,0, x_{5}, 0, x_{7}, \cdots\right) .
\end{aligned}
$$


Then $\sigma_{2}(A)=\emptyset$, thus $\varrho_{\tau}(A) \subseteq \varrho_{\mathrm{b}}(A) \cup \sigma_{2}(A)=\varrho(A)$. Also a straightforward calculation shows that

$$
\begin{gathered}
\sigma\left(\begin{array}{cc}
A & 0 \\
0 & B
\end{array}\right)=\sigma_{\mathrm{a}}\left(\begin{array}{cc}
A & 0 \\
0 & B
\end{array}\right)=\sigma\left(\begin{array}{cc}
A & C \\
0 & B
\end{array}\right)=\{0,1\}=\sigma_{\mathrm{a}}\left(\begin{array}{cc}
A & C \\
0 & B
\end{array}\right), \\
\sigma_{\mathrm{w}}\left(\begin{array}{cc}
A & 0 \\
0 & B
\end{array}\right)=\sigma_{\mathrm{ea}}\left(\begin{array}{cc}
A & 0 \\
0 & B
\end{array}\right)=\sigma_{\mathrm{w}}\left(\begin{array}{cc}
A & C \\
0 & B
\end{array}\right)=\sigma_{\mathrm{ea}}\left(\begin{array}{cc}
A & C \\
0 & B
\end{array}\right)=\{0,1\}, \\
\pi_{00}\left(\begin{array}{cc}
A & 0 \\
0 & B
\end{array}\right)=\pi_{00}^{\mathrm{a}}\left(\begin{array}{cc}
A & 0 \\
0 & B
\end{array}\right)=\emptyset,
\end{gathered}
$$

while

$$
\pi_{00}\left(\begin{array}{cc}
A & C \\
0 & B
\end{array}\right)=\pi_{00}^{\mathrm{a}}\left(\begin{array}{cc}
A & C \\
0 & B
\end{array}\right)=\{0\},
$$

which implies that Weyl's theorem and a-Weyl's theorem hold for $\left(\begin{array}{cc}A & 0 \\ 0 & B\end{array}\right)$, but fail for $\left(\begin{array}{cc}A & C \\ 0 & B\end{array}\right)$.

We now have:

Theorem 3.4. Let $A \in B(H)$ be such that iso $\sigma(A) \subseteq \varrho_{\tau}(A) \subseteq$ $\varrho_{\mathrm{b}}(A) \cup \sigma_{2}(A)$ and $B \in B(K)$.

(1) If Weyl's theorem holds for $\left(\begin{array}{cc}A & C_{0} \\ 0 & B\end{array}\right)$ for some $C_{0} \in B(K, H)$, then it holds for $\left(\begin{array}{cc}A & C \\ 0 & B\end{array}\right)$ for every $C \in B(K, H)$.

(2) If a-Weyl's theorem holds for $\left(\begin{array}{cc}A & C_{0} \\ 0 & B\end{array}\right)$ for some $C_{0} \in B(K, H)$, then it holds for $\left(\begin{array}{cc}A & C \\ 0 & B\end{array}\right)$ for every $C \in B(K, H)$.

Proof. The same argument as in the proof of Theorem 3.2 gives $\sigma\left(M_{C}\right) \backslash$ $\sigma_{\mathrm{w}}\left(M_{C}\right) \subseteq \pi_{00}\left(M_{C}\right)$ and $\sigma_{\mathrm{a}}\left(M_{C}\right) \backslash \sigma_{\mathrm{ea}}\left(M_{C}\right) \subseteq \pi_{00}^{\mathrm{a}}\left(M_{C}\right)$. For the reverse inclusion, first suppose $\lambda_{0} \in \pi_{00}\left(M_{C}\right)$. Then $0<\operatorname{dim} N\left(M_{C}-\lambda_{0} I\right)<\infty$ and there exists $\varepsilon>0$ such that $M_{C}-\lambda I$ is invertible if $0<\left|\lambda-\lambda_{0}\right|<\varepsilon$. It follows that $A-\lambda I$ is bounded from below and $B-\lambda I$ is surjective if $0<\left|\lambda-\lambda_{0}\right|<\varepsilon$. Then $\lambda \in \varrho_{\tau}(A) \subseteq \varrho_{\mathrm{b}}(A) \cup \sigma_{2}(A)$ and $\lambda \notin \sigma_{2}(A)$. Thus $A-\lambda I$ is Browder and hence $A-\lambda I$ is invertible because $A-\lambda I$ is injective. This implies $B-\lambda I$ is also invertible. Hence $\lambda_{0} \in$ iso $\sigma\left(M_{C_{0}}\right)$. We will show that $0<\operatorname{dim} N\left(M_{C_{0}}-\lambda_{0} I\right)<\infty$. First of all observe that there is a general inclusion

$$
N\left(M_{C}-\lambda_{0} I\right) \subseteq\left(A-\lambda_{0} I\right)^{-1}\left[C N\left(B-\lambda_{0} I\right)\right] \oplus N\left(B-\lambda_{0} I\right),
$$

which forces $N\left(A-\lambda_{0} I\right) \oplus N\left(B-\lambda_{0} I\right)$ to be nontrivial because otherwise $N\left(M_{C}-\lambda_{0} I\right)$ would be trivial, a contradiction. Now we must show that $N\left(A-\lambda_{0} I\right) \oplus N\left(B-\lambda_{0} I\right)$ is finite-dimensional. But since $N\left(A-\lambda_{0} I\right) \oplus\{0\} \subseteq$ $N\left(M_{C}-\lambda_{0} I\right)$, it follows that $\operatorname{dim} N\left(A-\lambda_{0} I\right)<\infty$. Thus we only need to prove that $\operatorname{dim} N\left(B-\lambda_{0} I\right)<\infty$. Suppose otherwise. Without loss of 
generality, suppose $\lambda_{0} \in \sigma(A)$. Then $\lambda_{0} \in$ iso $\sigma(A) \subseteq \varrho_{\tau}(A) \subseteq \varrho_{\mathrm{b}}(A) \cup \sigma_{2}(A)$ and $\lambda_{0} \notin \sigma_{2}(A)$, which means that $A-\lambda_{0} I$ is Browder. Now there are two cases to consider.

Suppose that $C N\left(B-\lambda_{0} I\right)$ is finite-dimensional. Then $N(C)$ must contain an orthonormal sequence $\left\{y_{i}\right\}$ in $N\left(B-\lambda_{0} I\right)$. But then $\left(\begin{array}{c}0 \\ y_{i}\end{array}\right) \in$ $N\left(M_{C}-\lambda_{0} I\right)$, which implies that $N\left(M_{C}-\lambda_{0} I\right)$ is infinite-dimensional, a contradiction.

Suppose $C N\left(B-\lambda_{0} I\right)$ is infinite-dimensional. Since $A-\lambda_{0} I$ is Browder, $R\left(A-\lambda_{0} I\right)^{\perp}$ is finite-dimensional. Therefore $C N\left(B-\lambda_{0} I\right) \cap R\left(A-\lambda_{0} I\right)$ is infinite-dimensional. Thus we can find an orthonormal sequence $\left\{y_{i}\right\}$ in $N\left(B-\lambda_{0} I\right)$ for which there exists a sequence $\left\{x_{i}\right\}$ in $H$ such that $\left(A-\lambda_{0} I\right) x_{i}=C y_{i}$ for each $i=1,2, \ldots$ Then $\left(\begin{array}{c}x_{i} \\ -y_{i}\end{array}\right) \in N\left(M_{C}-\lambda_{0} I\right)$, which implies that $N\left(M_{C}-\lambda_{0} I\right)$ is infinite-dimensional, a contradiction again.

From the preceding proof, we know that $0<\operatorname{dim}\left[N\left(A-\lambda_{0} I\right) \oplus N\left(B-\lambda_{0} I\right)\right]$ $<\infty$. The fact that $N\left(M_{C_{0}}-\lambda_{0} I\right) \subseteq\left(A-\lambda_{0} I\right)^{-1}\left[C N\left(B-\lambda_{0} I\right)\right] \oplus N\left(B-\lambda_{0} I\right)$ implies that $\operatorname{dim} N\left(M_{C_{0}}-\lambda_{0} I\right)<\infty$. If $N\left(M_{C_{0}}-\lambda_{0} I\right)=\{0\}$, then $N\left(A-\lambda_{0} I\right)$ $=\{0\}$, which means that $A-\lambda_{0} I$ is invertible. Thus $0<\operatorname{dim} N\left(B-\lambda_{0} I\right)$ $<\infty$. Let $y_{0} \in N\left(B-\lambda_{0} I\right)$ and $y_{0} \neq 0$. There exists $x_{0} \in H$ such that $\left(A-\lambda_{0} I\right) x_{0}=C_{0} y_{0}$, because $R\left(A-\lambda_{0} I\right)$ is surjective. Then $\left(\begin{array}{c}x_{0} \\ -y_{0}\end{array}\right) \in$ $N\left(M_{C_{0}}-\lambda_{0} I\right)$, a contradiction. Hense $\lambda_{0} \in \pi_{00}\left(M_{C_{0}}\right)$. Since Weyl's theorem holds for $M_{C_{0}}$, it follows that $M_{C_{0}}-\lambda_{0} I$ is Browder. As $A-\lambda_{0} I$ is Browder, so is $B-\lambda_{0} I$. Hence $M_{C}-\lambda_{0} I$ is Browder. Thus $\lambda_{0} \in \sigma\left(M_{C}\right) \backslash \sigma_{\mathrm{w}}\left(M_{C}\right)$, so $\sigma\left(M_{C}\right) \backslash \sigma_{\mathrm{w}}\left(M_{C}\right)=\pi_{00}\left(M_{C}\right)$, and hence Weyl's theorem holds for $M_{C}$ for each $C \in B(K, H)$.

If $\lambda_{0} \in \pi_{00}^{\mathrm{a}}\left(M_{C}\right)$, then $0<\operatorname{dim} N\left(M_{C}-\lambda_{0} I\right)<\infty$ and there exists $\varepsilon>0$ such that $M_{C}-\lambda I$ is bounded from below if $0<\left|\lambda-\lambda_{0}\right|<\varepsilon$. Then $A-\lambda I$ is bounded from below. Similarly to the preceding proof, we can show that $\lambda_{0} \in$ iso $\sigma_{\mathrm{a}}\left(M_{C_{0}}\right)$ and $A-\lambda_{0} I$ is Browder. Thus $\lambda_{0} \in \pi_{00}^{\mathrm{a}}\left(M_{C_{0}}\right)$. In a similar way, we deduce that a-Weyl's theorem holds for $M_{C}$ for any $C \in B(K, H)$.

The example in the Remark before Theorem 3.4 tells us that the condition iso $\sigma(A) \subseteq \varrho_{\tau}(A)$ is essential in Theorem 3.4.

Corollary 3.5. If iso $\sigma(A) \subseteq \varrho_{\tau}(A) \subseteq \varrho_{\mathrm{b}}(A) \cup \sigma_{2}(A)$ and iso $\sigma(B) \subseteq$ $\varrho_{\tau}(B) \subseteq \varrho_{\mathrm{b}}(B) \cup \sigma_{2}(B)$, then for every $C \in B(K, H)$, Weyl's theorem and $a$-Weyl's theorem hold for $f\left(M_{C}\right)$ for every $f \in H\left(M_{C}\right)$.

Proof. Suppose $f\left(M_{C}\right)-\mu I$ is Weyl and let $f\left(M_{C}\right)-\mu I=\left(M_{C}-\lambda_{1} I\right)^{n_{1}}$ $\cdots\left(M_{C}-\lambda_{k} I\right)^{n_{k}} g\left(M_{C}\right)$, where $\lambda_{i} \neq \lambda_{j}$ and $g\left(M_{C}\right)$ is invertible. Then $\sum_{i=1}^{k} \operatorname{ind}\left[\left(M_{C}-\lambda_{i} I\right)^{n_{i}}\right]=0$ and $M_{C}-\lambda_{i} I$ is Fredholm. This implies that both $A-\lambda_{i} I$ and $B-\lambda_{i} I$ are also Fredholm. Thus $\lambda_{i} \in \varrho_{\tau}(A) \subseteq \varrho_{\mathrm{b}}(A) \cup \sigma_{2}(A)$ and $\lambda_{i} \in \varrho_{\tau}(B) \subseteq \varrho_{\mathrm{b}}(B) \cup \sigma_{2}(B)$, so $\operatorname{ind}\left(A-\lambda_{i} I\right) \geq 0$ and $\operatorname{ind}\left(B-\lambda_{i} I\right) \geq 0$. Therefore $\operatorname{ind}\left(M_{C}-\lambda_{i} I\right) \geq 0$ for every $i=1, \ldots, k$, which implies that 
$\operatorname{ind}\left(M_{C}-\lambda_{i} I\right)=0$ because $\sum_{i=1}^{k} \operatorname{ind}\left[\left(M_{C}-\lambda_{i} I\right)^{n_{i}}\right]=0$. In this case, we must have $\lambda_{i} \in \varrho_{\mathrm{b}}(A) \cap \varrho_{\mathrm{b}}(B)$. Thus $M_{C}-\lambda_{i} I$ is Browder, and hence so is $f\left(M_{C}\right)-\mu I$, which means that $\sigma\left(f\left(M_{C}\right)\right) \backslash \sigma_{\mathrm{w}}\left(f\left(M_{C}\right)\right) \subseteq \pi_{00}\left(f\left(M_{C}\right)\right)$. For the converse, let $\mu \in \pi_{00}\left(f\left(M_{C}\right)\right)$ and

$$
f\left(M_{C}\right)-\mu I=\left(M_{C}-\lambda_{1} I\right)^{n_{1}} \cdots\left(M_{C}-\lambda_{k} I\right)^{n_{k}} g\left(M_{C}\right),
$$

where $\lambda_{i} \neq \lambda_{j}$ and $g\left(M_{C}\right)$ is invertible. Without loss of generality, we suppose $\lambda_{i} \in$ iso $\sigma\left(M_{C}\right)$. Then $\lambda_{i} \in$ iso $\sigma(A) \cup \varrho(A), \lambda_{i} \in$ iso $\sigma(B) \cup \varrho(B)$ and both $\operatorname{dim} N\left(A-\lambda_{i} I\right)<\infty$ and $\operatorname{dim} N\left(B-\lambda_{i} I\right)<\infty$. Using the assumption, we deduce that both $A-\lambda_{i} I$ and $B-\lambda_{i} I$ are Browder and hence so is $M_{C}-\lambda_{i} I$. This implies that $f\left(M_{C}\right)-\mu I$ is Browder, so $\pi_{00}\left(f\left(M_{C}\right)\right) \subseteq$ $\sigma\left(f\left(M_{C}\right)\right) \backslash \sigma_{\mathrm{w}}\left(f\left(M_{C}\right)\right)$. Therefore Weyl's theorem holds for $f\left(M_{C}\right)$ for every $f \in H\left(M_{C}\right)$. For a-Weyl's theorem, the proof is similar.

ExAmple. Let $A, B \in B\left(\ell_{2}\right)$ be defined by

$$
\begin{aligned}
& A\left(x_{1}, x_{2}, x_{3}, \ldots\right)=\left(x_{2}, x_{4}, x_{6}, \ldots\right), \\
& B\left(x_{1}, x_{2}, x_{3}, \cdots\right)=\left(0, x_{1}, 0, x_{2}, 0, x_{3}, 0, \ldots\right) .
\end{aligned}
$$

Then iso $\sigma(A)=\emptyset$ and $\sigma_{2}(A)=\sigma(A)$. Thus we have iso $\sigma(A) \subseteq \varrho_{\tau}(A) \subseteq$ $\varrho_{\mathrm{b}}(A) \cup \sigma_{2}(A)=\mathbb{C}$.

Using a straightforward calculation, we find that

$$
\begin{aligned}
\sigma\left(\begin{array}{cc}
A & 0 \\
0 & B
\end{array}\right) & =\sigma_{\mathrm{w}}\left(\begin{array}{cc}
A & 0 \\
0 & B
\end{array}\right)=\sigma_{\mathrm{b}}\left(\begin{array}{cc}
A & 0 \\
0 & B
\end{array}\right)=\mathbb{D}, \\
\sigma_{\mathrm{a}}\left(\begin{array}{cc}
A & 0 \\
0 & B
\end{array}\right) & =\sigma_{\mathrm{ea}}\left(\begin{array}{cc}
A & 0 \\
0 & B
\end{array}\right)=\sigma_{\mathrm{ab}}\left(\begin{array}{cc}
A & 0 \\
0 & B
\end{array}\right)=\mathbb{D},
\end{aligned}
$$

Thus both Weyl's theorem and a-Weyl's theorem hold for $\left(\begin{array}{cc}A & 0 \\ 0 & B\end{array}\right)$.

From Theorem 3.4, for each $C \in B\left(\ell_{2}, \ell_{2}\right)$, Weyl's theorem and a-Weyl's theorem hold for $M_{C}$.

Acknowledgments. We are grateful to the referees for helpful comments concerning this paper.

\section{References}

[1] P. Aiena and O. Monsalve, The single valued extension property and the generalized Kato decomposition property, Acta Sci. Math. (Szeged) 67 (2001), 461-477.

[2] X. H. Cao and B. Meng, Essential approximate point spectra and Weyl's theorem for operator matrices, J. Math. Anal. Appl. 304 (2005), 759-771.

[3] R. E. Curto and Y. M. Han, Weyl's theorem for algebraically paranormal operators, Integral Equations Operator Theory 47 (2003), 307-314.

[4] J. K. Finch, The single valued extension property on a Banach space, Pacific J. Math. 58 (1975), 61-69. 
[5] S. Grabiner, Uniform ascent and descent of bounded operators, J. Math. Soc. Japan 34 (1982), 317-337.

[6] Y. M. Han and S. V. Djordjević, a-Weyl's theorem for operator matrices, Proc. Amer. Math. Soc. 130 (2001), 715-722.

[7] R. Harte and W. Y. Lee, Another note on Weyl's theorem, Trans. Amer. Math. Soc. 349 (1997), 2115-2124.

[8] W. Y. Lee, Weyl's theorem for operator matrices, Integral Equations Operator Theory 32 (1998), 319-331.

[9] V. Rakočević, Operators obeying a-Weyl's theorem, Rev. Roumaine Math. Pures Appl. 34 (1989), 915-919.

College of Mathematics and Information Science

Shaanxi Normal University

Xi'an, 710062, People's Republic of China

E-mail: xiaohongcao@snnu.edu.cn

Received January 7, 2006

Revised version February 15, 2008 